Methods This is a prospective cohort study of 4200 moderate to severely injured patients at seven government hospitals in four LMICs (Cambodia, Kenya, Malaysia, and Vietnam). We administer a baseline and four follow-up surveys at home (at 1, 2, 4, and 12 months after discharge) to participants about their injury, functioning and disability, medical costs, employment, household composition and roles, as well as support systems (social \& economic).

Results The on-going study has enrolled 2293 individuals at the seven hospitals (Cambodia: 595, Kenya: 320, Malaysia: 284, and Vietnam: 1094). Participants are $72-84 \%$ male, with an average age of 30-42 years. The top cause of injury across all countries is road traffic injury $(47-72 \%)$. The second leading cause is falls (17-20\%), except for Kenya, where assaults rank second (20\%) and falls rank third (16\%). Changes in levels of disability, health care costs, productivity, household economic status, and roles of family members, as well as the respective influencing factors will be examined using marginal models with Generalised Estimating Equations (GEE) approach.

Conclusions Injury predominantly affects young males at their prime, having a significant impact not only on themselves, but their family as well as the society. This study will lead to a better understanding of the far reaching health, social, and economic impact of injuries. Data collected could be used to guide policy and programs in each of the implementing countries.

\section{FINANCIAL BURDEN OF INJURY AMONG A PROSPECTIVE COHORT OF INJURY PATIENTS IN MALAYSIA}

${ }^{1}$ Abdulgafoor M Bachani, ${ }^{1}$ Xiaoge Julia Zhang, ${ }^{2}$ Muhammad Fadhli Mohd Yusoff, ${ }^{2}$ Hamizatul Akmal binti Abd Hamid bin, ${ }^{2}$ Mohamad Naim Mohamad Rasidi, ${ }^{1}$ Adnan A Hyder. 'Johns Hopkins International Injury Research Unit, Johns Hopkins University Bloomberg School of Public Health, USA; ${ }^{2}$ Institute for Public Health, Malaysia Ministry of Health, Malaysia

\subsection{6/injuryprev-2016-042156.118}

Background Malaysia is rapidly developing and facing an increase in the burden of injuries, which disproportionally affect young and economically productive individuals. This study aims to understand injury-related economic burden on individuals and their families over time.

Methods We are recruiting a prospective cohort of 1200 moderately to severely injured patients from two government hospitals in Selangor and Kedah, Malaysia. We administer a baseline and four follow-up surveys $(1,2,4,12$ months after discharge) to subjects about socio-demographic characteristics, injury, family structure, and income changes. We obtain medical costs (direct costs) from medical bills and expenditure diaries. The outcome measures are direct and indirect costs.

Results Of 284 participants enrolled, $75 \%$ are male and $82 \%$ are Malay. Road traffic injury (70\%), fall (17\%) and blunt object injury $(17 \%)$ are the top three causes of injuries. On average, participants were hospitalised for 9.7 days (SD: 11.8), which was associated with productivity losses equivalent to RM411 (SD: 600) per person. Before injury, the average monthly income of participants was RM1304 (SD: 940), and the average medical care costs were RM156 (SD: 152). Income during hospitalisation was reduced in $24 \%$ of participants (RM534 on average). We will use marginal models with Generalised Estimating Equations to examine the trend of average costs. We will estimate average income changes by modelling the likelihood of having income reduction using logistic regression and size of income reduction using generalised linear regression.

Conclusions Injury brings about significant financial stress to individuals through direct medical costs, productivity loss and income reduction. This study will provide empirical evidence on the trend of injury costs and identify factors associated with high injury costs in Malaysia.

\section{Behavioural Science Research Informs Interventions to Reduce Child Injury - Panel Discussion}

\section{CLUSTER-RANDOMISED CONTROLLED TRIAL OF A FIRE SAFETY INJURY PREVENTION BRIEFING IN CHILDREN'S CENTRES}

${ }^{1}$ Toity Deave, ${ }^{2}$ Adrian Hawkins, ${ }^{3}$ Mike Hayes, ${ }^{4}$ Nicola Cooper, ${ }^{5}$ Carol Coupland ${ }^{6}$ Gosia Majsak-Newman, ${ }^{1}$ Trudy Goodenough, ${ }^{2}$ Elaine McColl, ${ }^{7}$ Richard Reading, ${ }^{5}$ Denise Kendrick. ${ }^{1}$ University of the West of England Bristol, UK; ${ }^{2}$ Newcastle University, Newcastle Upon Tyne, UK; ${ }^{3}$ Child Accident Prevention Trust, London, UK; ${ }^{4}$ University of Leicester, Leicester UK; ${ }^{5}$ University of Nottingham, Nottingham, UK; ${ }^{6}$ South Norfolk CCG, Norwich, UK; ${ }^{7}$ Norfolk and Norwich University Hospital, Norwich, UK

\subsection{6/injuryprev-2016-042156.119}

Background The UK has high mortality rates for fire-related deaths in children aged $0-14$ years with steep social gradients. Interventions to promote fire safety practices can be effective in reducing the risk of fire-related injury. We therefore developed an evidence-based fire safety intervention comprising an Injury Prevention Briefing (IPB), training and facilitation for use in children's centres.

Methods A cluster randomised controlled trial, with integrated qualitative study, was conducted across four study sites in England involving children's centres in disadvantaged areas; participants were staff and families attending those centres. Centres were stratified by study site and randomised within strata to one of three arms: IPB plus facilitation (IPB + ), IPB only, usual care. $\mathrm{IPB}+$ centres received initial training and facilitation at months 1, 3, and 8. Data collected comprised baseline and 12 months parent- and staff-completed questionnaires, home safety activity logs and staff interviews. The primary outcome was the proportion of families with a home fire-escape plan. Treatment arms were compared by using multilevel models to account for clustering by centre.

Results 1112 parents at 36 CCs participated. There was no significant effect of the intervention on family possession of fireescape plans (AOR IPB only vs. usual care: 0.93, 95\% CI: 0.58, 1.49; AOR IPB+ vs. usual care 1.41, 95\% CI: 0.91, 2.20). However, significantly more families in the intervention arms achieved more fire-escape planning behaviours (AOR IPB only vs. usual care: $2.56,95 \%$ CI: 01.38, 4.76; AOR IPB+ vs. usual care 1.78 , 95\% CI: 1.01, 3.15).

Conclusions Our study demonstrated that children's centres can deliver an injury prevention intervention to families in disadvantaged communities and achieve changes in home safety behaviours. 